\title{
Toileting behaviors and overactive bladder in patients with type 2 diabetes: a cross-sectional study in China
}

\author{
Dongjuan $\mathrm{Xu}^{1,2+}$, Ran Cheng ${ }^{1 \dagger}$, Aixia $\mathrm{Ma}^{3}$, Meng Zhao ${ }^{1}$ and Kefang Wang ${ }^{*^{*}}$ (D)
}

\begin{abstract}
Background: Overactive bladder is more prevalent in patients with type 2 diabetes than in those without diabetes. Unhealthy toileting behaviors may be associated with the development and worsening of overactive bladder symptoms. However, little is known about the relationships between toileting behaviors and overactive bladder in patients with diabetes. This study aimed to identify unhealthy toileting behaviors that patients with type 2 diabetes adopted to empty their bladders and investigate the relationships between toileting behaviors and overactive bladder.

Methods: Patients with type 2 diabetes from the endocrinology outpatient department of a hospital in China were recruited. The Toileting Behaviors-Women's Elimination Behavior and Overactive Bladder Symptom Score questionnaires were used to assess the patients' toileting behaviors and overactive bladder symptoms. A multivariate logistic regression model was used to explore the relationships between toileting behaviors and overactive bladder.

Results: Almost $14 \%$ of patients with diabetes had overactive bladder. The unhealthiest toileting behavior was premature voiding. In the multivariate logistic regression analysis, premature voiding $(\mathrm{OR}=1.286, p=0.016)$ and straining to void $(O R=1.243, p=0.026)$ were associated with overactive bladder. There was a greater likelihood of having overactive bladder when patients engaged in unhealthy toileting behaviors (premature voiding and straining to void).

Conclusions: Overactive bladder in patients with type 2 diabetes was more than twofold higher than that in the general population. Thus, overactive bladder is not just an inconsequential condition for patients with diabetes. Unhealthy toileting behaviors, e.g., premature voiding and straining to void, may contribute to the onset or worsening of overactive bladder in patients with diabetes. Identification and awareness of these modifiable behavioral factors during diabetes care is an essential component of primary prevention, alleviation, and management of overactive bladder symptoms.
\end{abstract}

Keywords: Overactive bladder, Diabetes mellitus, Toileting behaviors

\section{Background}

Overactive bladder, defined as "urinary urgency, usually accompanied by frequency and nocturia, with or without urgency urinary incontinence" [1], affects a large proportion of the general adult population. Approximately 455 million people worldwide (almost 11\%) experience overactive bladder, and the regional burden of overactive

\footnotetext{
* Correspondence: wangkf@sdu.edu.cn

Dongjuan Xu and Ran Cheng are joint first authors of this article.

${ }^{\dagger}$ Equal contributors

'School of Nursing, Shandong University, No.44, Wenhua Xi Road, Jinan,

Shandong 250012, China

Full list of author information is available at the end of the article
}

bladder is estimated to be greatest in Asia [2]. A recent large population-based survey conducted in China found that the prevalence of overactive bladder was 6\% [3]. Overactive bladder can have a substantial negative effect on patients' quality of life specifically in terms of physical and psychological well-being, social interactions, work productivity, and sexual health $[4,5]$. In addition, the direct and indirect costs of overactive bladder could seriously aggravate the economic burden on individuals and healthcare systems [6].

Unhealthy toileting behaviors may be associated with the development and worsening of overactive bladder 
symptoms. Individuals may empty bladder more frequently than necessary, based on the misconception that frequent voiding avoids bladder incontinence episodes [7]. However, too frequent voiding only signal an urge to empty the bladder despite a smaller volume of urine [8], which can precipitate or exacerbate bladder dysfunction [9]. Straining to empty the bladder, which involves an abdominal muscle contraction, could increase the peak flow and mean flow rates, as well as decrease the total voiding time [10]. Individuals who use straining to start voiding before the initiation of the micturition reflex may be more likely to develop incontinence and voiding dysfunction [11]. Toileting behavior is a comprehensive concept that includes voiding place, time, position, and style [12]; however, most recent studies on toileting behavior and overactive bladder focused only on one or some aspects of toileting behavior [13, 14]. Previously, Wan et al. examined the relationship between toileting behaviors and lower urinary tract symptoms among female nurses and found that three unhealthy toileting behaviors (i.e., premature voiding, delayed voiding, and straining to void) were significantly associated with lower urinary tract symptoms, while no significant association with voiding place or position preference was observed [15]. However, little is known about the relationships between toileting behaviors and overactive bladder in other populations.

Diabetes mellitus has been related to an earlier onset and increased bladder dysfunction severity [16]. Overactive bladder is more prevalent in patients with type 2 diabetes than in the general population [17, 18]. Moreover, higher glycosylated hemoglobin levels increased the risk of overactive bladder in patients with diabetes [19]. Besides diabetes-related factors, a variety of factors were associated with overactive bladder, including age [3, 19], gender [20], marital status [3], fluid intake [21], smoking [22], alcohol drinking [3], physical exercise [23], history of urinary tract infection [24], constipation [25], hypertension/heart failure [26], and obesity [20].

Because of its high prevalence, overactive bladder is an important therapeutic target in patients with type 2 diabetes. Available evidence suggests that behavioral interventions, such as bladder training [27], pelvic floor muscle training and exercise, and urge suppression techniques [28], are effective in extending voiding interval and alleviating urgency and incontinence. Behavioral interventions may also increase the efficacy of pharmacologic treatment for overactive bladder [9]. If unhealthy toileting behaviors can contribute to overactive bladder symptoms, identifying and modifying these unhealthy behaviors, accompanied by other behavioral and pharmacologic treatments, help prevent, eliminate, alleviate, and manage overactive bladder symptoms in patients with type 2 diabetes. Therefore, the purposes of this study were (1) to investigate the unhealthy toileting behaviors that patients with diabetes adopted to empty their bladders and (2) to identify the relationships between toileting behaviors and overactive bladder in patients with type 2 diabetes.

\section{Methods}

\section{Study design and data collection}

This is a cross-sectional study with convenience sampling. From May to August 2014, we recruited participants from the endocrinology outpatient department in one of the largest hospitals in Jinan, the capital city of Shandong Province, China. The inclusion criteria were as follows: (1) $\geq 18$ years old, (2) diagnosed as having type 2 diabetes, and (3) willing to participate in the study and capable of understanding study procedure and questions. The exclusion criteria were the following: (1) newonset neurological disorders (such as stroke, spinal cord damage, Parkinson's disease, and multiple sclerosis), (2) urinary tract infections within the last month of the survey, (3) pelvic organ prolapse, and (4) history of bladder surgery.

The Institutional Review Board of Shandong University approved this study. A self-administered pencil-andpaper survey was used to collect data. Before the survey, trained graduate students obtained written informed consent from each patient with diabetes. The survey was completed anonymously and the patients were assured that their responses would be kept confidential.

\section{Assessment}

We obtained the patients' sociodemographic characteristics data, including age, sex, race/ethnicity, education, marital status, living area, and income, and assessed lifestyle-related characteristics, including smoking, alcohol use, tea drinking, fluid intake, and physical exercise. We also collected health-related data consisting of waist circumference, hip circumference, urinary tract infection history, comorbidity, diabetes mellitus duration, and microvascular complications of diabetes (peripheral neuropathy and retinopathy). Moreover, the Charlson comorbidity index (CCI) was used to measure a range of comorbid conditions, such as hypertension, congestive heart failure, or cancer (a total of 20 conditions) [29]. Since all patients in this study had diabetes, diabetes was excluded from the calculation of CCI. Each condition was assigned a weight of $1,2,3$, or 6 , depending on the risk of mortality [29]. The total score, which is the sum of all weighted conditions, was used to predict mortality.

We used the validated Toileting Behaviors-Women's Elimination Behavior (TB-WEB) scale (Chinese version) to evaluate the participants' toileting behaviors [30]. This 17-item scale contains five domains: place preference for voiding, premature voiding, delayed voiding, straining to 
void, and position preference for voiding. Since position preference for voiding (e.g., "crouching or hovering to empty bladder when not at home") is specific for the female population, we did not include this domain in the study. Detailed descriptions of each item are provided in Table 3. The TB-WEB used a five-point Likert-type scale (from 1 = never to 5 = always) to assess how often patients with diabetes adopted a behavior. An average score was calculated for each domain, and a higher score corresponding to unhealthier toileting behavior. The 4domain TB-WEB has been validated in male patients [31], and the Cronbach's alpha coefficient of the TBWEB scale was 0.71 in this study.

We used the Chinese version of Overactive Bladder Symptom Score (OABSS) questionnaire to evaluate overactive bladder symptoms in the past week. OABSS is a reliable and valid questionnaire designed to quantify the four symptoms of overactive bladder: daytime frequency $(0-2)$, nighttime frequency (0-3), urgency (0-5), and urgency incontinence $(0-5)$ [32]. The total score ranges from 0 to 15, and a higher score represents more severe overactive bladder symptoms. The Chinese version of the OABSS scale has been developed and validated in the Chinese population with good reliability and validity [33]. Here, overactive bladder means an urgency symptom score $\geq 2$ and a total score $\geq 3$.

\section{Statistical analysis}

We used descriptive statistics (frequency and percentage for categorical variables, mean and standard deviation for continuous variables) to describe the characteristics of patients with type 2 diabetes. We performed Student's $t$-tests to compare the differences of each toileting behavior between patients without overactive bladder and those with overactive bladder. We also used a multivariate logistic regression model to explore the relationships between toileting behaviors and overactive bladder, after adjusting for age, sex, race/ethnicity, education, marital status, living area, income, smoking, alcohol use, tea drinking, fluid intake per day, physical exercise, waist-tohip ratio, urinary tract infection history, CCI, diabetes mellitus duration, and microvascular complications of diabetes (peripheral neuropathy and retinopathy). We performed all statistical analyses using Stata (Version 14.1; StataCorp, College Station, TX). Statistical significance was accepted at $p<0.05$.

\section{Results}

Among the 1025 eligible patients with type 2 diabetes, seven patients were excluded from the analysis because they omitted four or more survey items. The mean age was approximately 59 years (range 20-86 years), and half of the participants were female. The patients had diabetes for about 9 years on average, and more than half (52\%) had diabetic peripheral neuropathy or retinopathy. Nearly $14 \%$ of the patients had overactive bladder. Among the overactive bladder patients, approximately $57 \%$ had wet overactive bladder and $43 \%$ had dry overactive bladder. The patient characteristics are presented in Table 1.

The unhealthiest toileting behavior among patients with diabetes was premature voiding, followed by place preference for voiding, delayed voiding, and straining to void (Table 2). Premature voiding and straining to void were significantly unhealthier in patients with overactive bladder than in those without overactive bladder, as indicated by Student's $t$-tests. As shown in Table 3, about half of the patients often or always emptied their bladders with little or no need to urinate before sleep (57\%) or before leaving home (47\%). Approximately $36 \%$ of the patients often or always avoided using toilets at someone else's house. With regard to public toilets, $26 \%$ of the patients were often or always worried about the cleanness and $24 \%$ of the patients avoided using them. More than $15 \%$ of patients often or always waited more than $4 \mathrm{~h}$ to urinate at work, and $13 \%$ of the patients often or always pushed down or strained to finish emptying their bladder.

After adjusting for control variables, positive associations between unhealthy toileting behaviors (premature voiding and staining to void) and overactive bladder were found among patients with diabetes (Table 4). The more the patients empty their bladder with little or no need to void and pushed down or strained to void, the greater the likelihood of having overactive bladder. There were no significant associations between toileting behaviors (place preference for voiding and delayed voiding) and overactive bladder. In addition, age, waistto-hip ratio, CCI, and diabetic peripheral neuropathy or retinopathy were positively associated with overactive bladder, while female and married patients with diabetes were less likely to have overactive bladder.

\section{Discussion}

A large sample survey of patients with type 2 diabetes was conducted to identify unhealthy toileting behaviors and further investigate their relationships with overactive bladder. The present study adds to the small body of work that extends research focused on only one or some aspects of toileting behavior to comprehensively examine it using a valid instrument. We found that the prevalence of overactive bladder in patients with diabetes was nearly $14 \%$, which is more than twofold higher than that in the general population in China [3]. Moreover, the more the patients with diabetes engage in unhealthy toileting behaviors (e.g., premature voiding and straining to void), the greater the likelihood of having overactive bladder. Thus, our findings provide significant 
Table 1 Characteristics of patients with type 2 diabetes mellitus $(n=1018)$

\begin{tabular}{ll}
\hline Variables & Mean \pm SD or $n(\%)$ \\
\hline Age (years) & $59.1 \pm 11.7$ \\
Sex & \\
$\quad$ Female & $509(50.0)$ \\
$\quad$ Male & $509(50.0)$ \\
Race/ethnicity & \\
$\quad$ Han & $981(96.4)$
\end{tabular}

Other

37 (3.6)

Education

Elementary school or lower

$228(22.4)$

Middle school

$232(22.8)$

High school

306 (30.1)

College or higher

$252(24.7)$

Marital status

Married

$920(90.4)$

Single/divorced/widowed

Living area

Urban

Rural

Income (RMB (USD)/month)

$\leq 3000$ (451)

$620(60.9)$

$>3000$ (451)

398 (39.1)

Smoker

Yes

345 (33.9)

No

$673(66.1)$

Alcohol drinker

Yes

308 (30.3)

No

$710(69.7)$

Tea drinker

Yes

732 (71.9)

No

$286(28.1)$

Fluid intake (ml/day)

$\leq 2500$

$546(53.6)$

$>2500$

$472(46.4)$

Exercise

Yes

No

Waist-to-hip ratio (WHR)

Urinary tract infection history ${ }^{a}$

$$
\text { Yes }
$$

No

Charlson comorbidity index (CCl)

Diabetes mellitus duration (years)
Table 1 Characteristics of patients with type 2 diabetes mellitus $(n=1018)$ (Continued)

\begin{tabular}{ll}
\hline $\begin{array}{l}\text { Diabetic peripheral neuropathy or retinopathy } \\
\text { Yes }\end{array}$ & $533(52.4)$ \\
No & $485(47.6)$ \\
overactive bladder & \\
No overactive bladder & $878(86.3)$ \\
Dry overactive bladder & $60(5.9)$ \\
Wet overactive bladder & $80(7.9)$
\end{tabular}

SD standard deviation, RMB Chinese Yuan, USD United States dollar

${ }^{\text {aP }}$ Patients with type 2 diabetes who had a urinary tract infection at least one month before the survey

implications in the prevention, alleviation, and management of overactive bladder symptoms during diabetes care.

The findings indicated that the most common unhealthy toileting behavior among patients with diabetes was premature voiding. Approximately half of them often or always emptied their bladder with little or no need to void before sleep or before leaving home. Moreover, premature voiding was significantly associated with overactive bladder in patients with diabetes, which is in line with the finding by Wan et al., who found a positive association between premature voiding and lower urinary tract symptoms in female nurses [15]. In addition, too frequent voiding with little or no need to urinate may have no harmful effects in the short term; however, engaging in this behavior leads to increased bladder sensitivity to lower volumes of urine, reduced bladder capacity, and eventually detrusor instability in the long term $[8,9]$.

We also found that straining to void, although the least common unhealthy toileting behavior, had a positive association with overactive bladder in patients with type 2 diabetes. This finding confirms that from a study of female nurses with regard to straining to void and lower urinary tract symptoms [15]. Pushing down or straining was often or always employed by patients with diabetes to begin urinating (7.0\%), keep urine flowing (6.4\%), empty bladder more quickly (5.7\%), and finish emptying bladder (13.4\%). Abdominal straining to void is considered unhealthy by researchers because of its association with poor outcomes, such as fecal and/or urinary incontinence [34], dysfunctional voiding [13], and prolonged postoperative catheterization [35]. Pauwels et al. indicated that if straining was used to begin voiding before the initiation of the micturition reflex and as the only way to empty bladder, voiding problems and incontinence are more likely to occur [11].

In this study, delayed voiding and place preference for voiding were not significantly associated with overactive bladder in patients with diabetes; the patients with 
Table 2 Comparison of toileting behaviors between patients with diabetes with and without overactive bladder

\begin{tabular}{|c|c|c|c|c|}
\hline Toileting behavior & $\begin{array}{l}\text { Overall sample } \\
(n=1018)\end{array}$ & $\begin{array}{l}\text { No OAB } \\
(n=878)\end{array}$ & $\begin{array}{l}\text { OAB } \\
(n=140)\end{array}$ & $p$ value \\
\hline Premature voiding & $2.41 \pm 1.00$ & $2.37 \pm 0.98$ & $2.68 \pm 1.04$ & 0.001 \\
\hline Place preference for voiding & $2.18 \pm 1.14$ & $2.18 \pm 1.14$ & $2.14 \pm 1.13$ & 0.710 \\
\hline Delayed voiding & $2.06 \pm 0.74$ & $2.05 \pm 0.73$ & $2.15 \pm 0.77$ & 0.142 \\
\hline Straining to void & $1.49 \pm 0.88$ & $1.45 \pm 0.84$ & $1.74 \pm 1.04$ & 0.002 \\
\hline
\end{tabular}

The numbers are expressed as mean \pm standard deviation $O A B$ overactive bladder

overactive bladder had almost the same scores for the two unhealthy toileting behaviors as those of patients without overactive bladder. Nevertheless, delayed voiding and place preference for voiding were common in patients with diabetes. Moreover, suppressing the urge to void may have no detrimental effect in the short term; however, long-term suppression may threaten bladder's health [14]. Avoiding using public toilets or toilets at someone else's house may lead to premature or delayed voiding [36]. Thus, educating patients to adopt healthy toileting behaviors to improve and maintain their optimal bladder health is essential.

Besides unhealthy toileting behaviors, we found another significant modifiable factor associated with overactive bladder in patients with diabetes, i.e., waist-to-hip ratio. Waist-to-hip ratio, as a measure of obesity or health, has been shown to be a better predictor of cardiovascular disease [37], diabetes [38], and hypercholesterolemia [39] compared with body mass index and waist circumstance. Our finding is similar to studies that showed obesity to be a risk factor of overactive bladder and weight loss to be related to improvements in incontinence status $[20,40]$. Weight loss has a beneficial effect on general health and quality of life; thus, it should be encouraged universally. In addition, diabetic peripheral neuropathy or retinopathy, which is the consequence of poor blood glucose control, was found to be associated with overactive bladder. Similarly, Chiu et al. showed that the increased risk of overactive bladder in patients with diabetes could be attributed to higher glycosylated hemoglobin levels [19]. Furthermore, overactive bladder may also result from a number of conditions or comorbidities, such as hypertension, heart failure, cerebrovascular disease, dementia, and renal diseases [26], which

Table 3 Toileting behaviors among patients with type 2 diabetes mellitus ( $n=1018$ )

\begin{tabular}{|c|c|c|c|}
\hline Behavior & $\begin{array}{l}\text { Never/Rarely } \\
n(\%)\end{array}$ & $\begin{array}{l}\text { Sometimes } \\
n(\%)\end{array}$ & $\begin{array}{l}\text { Often/Always } \\
n(\%)\end{array}$ \\
\hline \multicolumn{4}{|l|}{ Premature voiding } \\
\hline Empty bladder with little or no need to urinate before sleep & $348(34.2)$ & $89(8.7)$ & $581(57.1)$ \\
\hline Empty bladder with little or no need to urinate before leaving home & $412(40.5)$ & $131(12.9)$ & $475(46.7)$ \\
\hline Empty bladder with little or no need to urinate "just in case" & $701(68.9)$ & $132(13.0)$ & $185(18.2)$ \\
\hline Empty bladder with little or no need to urinate at home & $938(92.1)$ & $54(5.3)$ & $26(2.6)$ \\
\hline \multicolumn{4}{|l|}{ Place preference for voiding } \\
\hline Avoid using toilets at someone else's house & $588(57.8)$ & $63(6.2)$ & $367(36.1)$ \\
\hline Worry about cleanness of public toilets & $650(63.9)$ & $104(10.2)$ & $264(25.9)$ \\
\hline Avoid using public toilets & $690(67.8)$ & $82(8.1)$ & $246(24.2)$ \\
\hline Hold urine until getting home & $705(69.3)$ & $164(16.1)$ & $149(14.6)$ \\
\hline \multicolumn{4}{|l|}{ Delay voiding } \\
\hline Wait too long $(>4 \mathrm{~h})$ to urinate at work & $690(67.8)$ & $172(16.9)$ & $156(15.3)$ \\
\hline Delay emptying bladder when busy & $572(56.2)$ & $326(32.0)$ & $120(11.8)$ \\
\hline Wait to empty bladder until unable to hold & $788(77.4)$ & $152(14.9)$ & $78(7.7)$ \\
\hline \multicolumn{4}{|l|}{ Straining to void } \\
\hline Push down or strain to finish emptying bladder & $774(76.0)$ & $108(10.6)$ & $136(13.4)$ \\
\hline Push down or strain to begin urinating & $883(86.7)$ & $64(6.3)$ & $71(7.0)$ \\
\hline Push down or strain to keep urine flowing & $885(86.9)$ & $68(6.7)$ & $65(6.4)$ \\
\hline Push down or strain to empty bladder more quickly & $880(86.4)$ & $80(7.9)$ & $58(5.7)$ \\
\hline
\end{tabular}


Table 4 The relationships between toileting behaviors and overactive bladder among patients with type 2 diabetes mellitus $(n=1018)$

\begin{tabular}{|c|c|c|c|}
\hline Variables & OR $(95 \% \mathrm{Cl})$ & SE & $p$ value \\
\hline \multicolumn{4}{|l|}{ Toileting behavior } \\
\hline Premature voiding & $1.286(1.048-1.579)$ & 0.135 & 0.016 \\
\hline Straining to void & $1.243(1.026-1.506)$ & 0.122 & 0.026 \\
\hline Delayed voiding & $1.259(0.969-1.636)$ & 0.168 & 0.084 \\
\hline Place preference for voiding & $0.883(0.735-1.062)$ & 0.083 & 0.188 \\
\hline Age & $1.027(1.006-1.049)$ & 0.011 & 0.013 \\
\hline Sex (female vs. male) & $0.484(0.274-0.854)$ & 0.140 & 0.012 \\
\hline Race/Ethnicity (Han vs. other) & $4.624(0.979-21.832)$ & 3.662 & 0.053 \\
\hline \multicolumn{4}{|c|}{ Education (ref. = elementary school or lower) } \\
\hline Middle school & $0.831(0.471-1.466)$ & 0.241 & 0.522 \\
\hline High school & $0.638(0.360-1.130)$ & 0.186 & 0.124 \\
\hline College or higher & $0.584(0.308-1.108)$ & 0.191 & 0.100 \\
\hline $\begin{array}{l}\text { Marital status (married vs. } \\
\text { unmarried) }\end{array}$ & $0.513(0.285-0.922)$ & 0.154 & 0.026 \\
\hline Living area (urban vs. rural) & $1.219(0.706-2.107)$ & 0.340 & 0.477 \\
\hline Income (>3000 vs. $\leq 3000$ ) & $0.807(0.523-1.245)$ & 0.178 & 0.331 \\
\hline Smoker & $1.105(0.659-1.855)$ & 0.292 & 0.705 \\
\hline Alcohol drinker & $0.638(0.379-1.073)$ & 0.169 & 0.090 \\
\hline Tea drinker & $1.215(0.781-1.890)$ & 0.274 & 0.388 \\
\hline Fluid intake (>2500 vs. $\leq 2500$ ) & $1.070(0.726-1.575)$ & 0.211 & 0.734 \\
\hline Exercise & $0.823(0.549-1.233)$ & 0.170 & 0.345 \\
\hline Waist-to-hip ratio (WHR) ${ }^{\text {a }}$ & $1.266(1.033-1.552)$ & 0.131 & 0.023 \\
\hline Urinary tract infection history ${ }^{b}$ & $0.966(0.496-1.880)$ & 0.328 & 0.919 \\
\hline $\begin{array}{l}\text { Charlson comorbidity } \\
\text { index (CCl) }\end{array}$ & $1.183(1.001-1.397)$ & 0.101 & 0.049 \\
\hline Diabetes mellitus duration & $0.984(0.955-1.013)$ & 0.015 & 0.270 \\
\hline $\begin{array}{l}\text { Diabetic peripheral neuropathy } \\
\text { or retinopathy }\end{array}$ & $1.506(1.002-2.264)$ & 0.313 & 0.049 \\
\hline
\end{tabular}

$O R$ odds ratio, $\mathrm{Cl}$ confidence interval, $S E$ standard error a Standardized waist-to-hip ratio (WHR) was used in the analysis ${ }^{\mathrm{b}}$ Patients with type 2 diabetes who had a urinary tract infection at least one month before the survey

supports our result that higher CCI was associated with increased risk of overactive bladder. Our results are consistent with a previous report that overactive bladder increased with age and was more prevalent in men than in women [20].

This study has several limitations. First, we recruited patients in one hospital using a convenience sampling method. Thus, the generalizability of our findings may be limited. To address this, we surveyed a large sample of 1025 patients with diabetes with $<1 \%$ missing data. Second, because of the cross-sectional design of the survey, a causal relationship between unhealthy toileting behaviors and overactive bladder could not be established. Moreover, in cross-sectional studies, associations could be reversed. For example, patients might adopt certain toileting behaviors to cope with overactive bladder symptoms. However, this may not be the case in our study because we surveyed unhealthy toileting behaviors and later overactive bladder symptoms. Exploring toileting behavior changes after having overactive bladder symptoms and determining whether these changes further worsen or relieve overactive bladder symptoms would be interesting. Third, our findings may be influenced by uncontrolled confounders, such as constipation, benign prostatic hyperplasia, and vaginal or cesarean delivery. Future studies should include these variables in the survey and explore whether the associations between toileting behaviors and overactive bladder vary by gender.

\section{Conclusions}

Overactive bladder in patients with type 2 diabetes was more than twofold higher than that in the general population. Thus, overactive bladder is not just an inconsequential condition for patients with diabetes. Furthermore, in patients with diabetes, preserving bladder health is particularly necessary and unhealthy toileting behaviors, especially premature voiding and straining to void, may contribute to the onset or worsening of overactive bladder. Identification and awareness of modifiable behavioral factors during diabetes care is an essential component of primary prevention, alleviation, and management of overactive bladder symptoms.

\section{Abbreviations}

CCl: Charlson comorbidity index; OAB: Overactive bladder; OABSS: Overactive Bladder Symptom Score; TB-WEB: Toileting Behaviors-Women's Elimination Behavior

Acknowledgements

The authors thank all of the patients for their participation.

Funding

This work was supported by a grant from the Natural Science Foundation of Shandong Province (ZR2015HM033). The funders had no role in study design, data collection, data analysis, manuscript preparation, and publication decisions.

Availability of data and materials

The datasets used and/or analysed during the current study are available from the corresponding author on reasonable request.

Authors' contributions

KFW designed the study. RC, AXM and MZ collected the data. DJX analyzed the data. DJX and RC drafted the manuscript. KFW supervised the study and made critical revisions to the paper for important intellectual content. All authors read and approved the final manuscript.

Competing interests

The authors declare that they have no competing interests.

Consent for publication

Not applicable. 


\section{Ethics approval and consent to participate}

All subjects signed an informed consent. The Shandong University School of Nursing Institutional Review Board approved this study. The IRB approval number is EA2013033.

\section{Publisher's Note}

Springer Nature remains neutral with regard to jurisdictional claims in published maps and institutional affiliations.

\section{Author details}

${ }^{1}$ School of Nursing, Shandong University, No.44, Wenhua Xi Road, Jinan, Shandong 250012, China. ${ }^{2}$ School of Nursing, Purdue University, West Lafayette, Indiana 47907, USA. ${ }^{3}$ Department of endocrinology, Qilu Hospital of Shandong University, Jinan, Shandong 250012, China.

Received: 29 March 2017 Accepted: 5 June 2017

Published online: 13 June 2017

\section{References}

1. Haylen BT, de Ridder D, Freeman RM, et al. An international Urogynecological association (IUGA)/international Continence society (ICS) joint report on the terminology for female pelvic floor dysfunction. Neurourol Urodyn. 2010;29:4-20.

2. Irwin DE, Kopp ZS, Agatep B, et al. Worldwide prevalence estimates of lower urinary tract symptoms, overactive bladder, urinary incontinence and bladder outlet obstruction. BJU Int. 2011;108:1132-8.

3. Wang $Y, X u K, H u H$, et al. Prevalence, risk factors, and impact on health related quality of life of overactive bladder in China. Neurourol Urodyn. 2011:30:1448-55.

4. Irwin DE, Milsom I, Kopp Z, et al. Impact of overactive bladder symptoms on employment, social interactions and emotional well-being in six European countries. BJU Int. 2006;97:96-100

5. Coyne KS, Sexton CC, Thompson C, et al. The impact of OAB on sexual health in men and women: results from EpiLUTS. J Sex Med. 2011;8: 1603-15.

6. Ganz ML, Smalarz AM, Krupski TL, et al. Economic costs of overactive bladder in the United States. Urology. 2010:75:526-32.

7. Naoemova I, De Wachter S, Wyndaele JJ. Comparison of sensation-related voiding patterns between continent and incontinent women: a study with a 3-day sensation-related bladder diary (SR-BD). Neurourol Urodyn. 2008;27: $511-4$.

8. Sampselle CM. Teaching women to use a voiding diary. Am J Nurs. 2003; 103:62-4.

9. Burgio KL. Influence of behavior modification on overactive bladder Urology. 2002;60:72-6.

10. Devreese AM, Nuyens G, Staes F, et al. Do posture and straining influence urinary-flow parameters in normal women? Neurourol Urodyn. 2000;19:3-8.

11. Pauwels E, De Laet K, De Wachter S, et al. Healthy, middle-aged, history-free, continent women-do they strain to void? J Urol. 2006;175:1403-7.

12. Wang $\mathrm{K}$, Palmer $\mathrm{MH}$. Development and validation of an instrument to assess women's toileting behavior related to urinary elimination: preliminary results. Nurs Res. 2011;60:158-64.

13. Carlson KV, Rome S, Nitti WW. Dysfunctional voiding in women. J Urol. 2001; 165:143-7.

14. Zhang C, Hai T, Yu L, et al. Association between occupational stress and risk of overactive bladder and other lower urinary tract symptoms: a crosssectional study of female nurses in China. Neurourol Urodyn. 2013;32: 254-60

15. Wan $X$, Wu $C, X u D$, et al. Toileting behaviours and lower urinary tract symptoms among female nurses: a cross-sectional questionnaire survey. Int J Nurs Stud. 2017:65:1-7.

16. Karoli R, Bhat S, Fatima J, et al. A study of bladder dysfunction in women with type 2 diabetes mellitus. Indian J Endocrinol Metab. 2014;18:552-7.

17. Ikeda M, Nozawa K. Prevalence of overactive bladder and its related factors in Japanese patients with diabetes mellitus. Endocr J. 2015;62:847-54.

18. Lawrence JM, Lukacz ES, Liu IL, et al. Pelvic floor disorders, diabetes, and obesity in women: findings from the Kaiser Permanente Continence associated risk epidemiology study. Diabetes Care. 2007:30:2536-41.

19. Chiu AF, Huang MH, Wang CC, et al. Higher glycosylated hemoglobin levels increase the risk of overactive bladder syndrome in patients with type 2 diabetes mellitus. Int J Urol. 2012;19:995-1001.
20. Wen JG, Li JS, Wang ZM, et al. The prevalence and risk factors of OAB in middle-aged and old people in China. Neurourol Urodyn. 2014;33:387-91.

21. Callan L, Thompson DL, Netsch D. Does increasing or decreasing the daily intake of water/fluid by adults affect overactive bladder symptoms? J Wound Ostomy Continence Nurs. 2015;42:614-20.

22. de Boer TA, Slieker-ten Hove MC, Burger CW, et al. The prevalence and risk factors of overactive bladder symptoms and its relation to pelvic organ prolapse symptoms in a general female population. Int Urogynecol J. 2011; 22:569-75.

23. Ko IG, Lim MH, Choi PB, et al. Effect of long-term exercise on voiding functions in obese elderly women. Int Neurourol J. 2013:17:130-8.

24. Chung JM, Lee SD, Kang DI, et al. Prevalence and associated factors of overactive bladder in Korean children 5-13 years old: a nationwide multicenter study. Urology. 2009;73:63-7.

25. Wu JW, Xing YR, Wen YB, et al. Prevalence of Spina bifida Occulta and its relationship with overactive bladder in middle-aged and elderly Chinese people. Int Neurourol J. 2016;20:151-8.

26. Asche CV, Kim J, Kulkarni AS, et al. Presence of central nervous system, cardiovascular and overall co-morbidity burden in patients with overactive bladder disorder in a real-world setting. BJU Int. 2012;109:572-80.

27. Christofi N, Hextall A. An evidence-based approach to lifestyle interventions in urogynaecology. Menopause Int. 2007:13:154-8.

28. Burgio KL, Goode PS, Johnson TM, et al. Behavioral versus drug treatment for overactive bladder in men: the male overactive bladder treatment in veterans (MOTIVE) trial. J Am Geriatr Soc. 2011;59:2209-16.

29. Charlson ME, Pompei $\mathrm{P}$, Ales $\mathrm{KL}$, et al. A new method of classifying prognostic comorbidity in longitudinal studies: development and validation. J Chronic Dis. 1987:40:373-83.

30. Wan X, Zhang Y, Liu Y, et al. Reliability and validity of the Chinese version of Women's toileting behavior scale among female nurses. Chin J Nurs. 2014: 49:782-5.

31. Cheng R, Gao J, Chen LQ, et al. Reliability and validity of the toileting behavior scale in men with diabetes mellitus. J Nurs. 2016:23:8-11.

32. Homma Y, Yoshida M, Seki N, et al. Symptom assessment tool for overactive bladder syndrome- overactive bladder symptom score. Urology. 2006:68: $318-23$

33. Hung MJ, Chou CL, Yen TW, et al. Development and validation of the Chinese overactive bladder symptom score for assessing overactive bladder syndrome in a RESORT study. J Formos Med Assoc. 2013;112:276-82.

34. Lacima G, Espuna M, Pera M, et al. Clinical, urodynamic, and manometric findings in women with combined fecal and urinary incontinence. Neurourol Urodyn. 2002;21:464-9.

35. Bhatia NN, Bergman A. Urodynamic predictability of voiding following incontinence surgery. Obstet Gynecol. 1984;63:85-91.

36. Xu D, Chen L, Wan X, et al. Toileting behaviour and related health beliefs among Chinese female nurses. Int J Clin Pract. 2016;70:416-23.

37. de Koning L, Merchant AT, Pogue J, et al. Waist circumference and waist-tohip ratio as predictors of cardiovascular events: meta-regression analysis of prospective studies. Eur Heart J. 2007;28:850-6.

38. Motamed N, Rabiee B, Keyvani $\mathrm{H}$, et al. The best obesity indices to discriminate type 2 diabetes mellitus. Metab Syndr Relat Disord. 2016;14 249-53.

39. Haregu TN, Oti S, Egondi T, et al. Measurement of overweight and obesity an urban slum setting in sub-Saharan Africa: a comparison of four anthropometric indices. BMC Obes. 2016:3:46.

40. Subak LL, King WC, Belle SH, et al. Urinary incontinence before and after bariatric surgery. JAMA Intern Med. 2015;175:1378-87. 